\title{
Random Fixed Point Theorems and Applications to Random First- Order Vector-Valued Differential Equations
}

\author{
Adil El-Ghabi $(\mathbb{D}$, Abdelmjid Khchine $\mathbb{D}$, and Mohamed Aziz Taoudi $(\mathbb{D}$ \\ Cadi Ayyad University, National School of Applied Science, Marrakech, Morocco \\ Correspondence should be addressed to Adil El-Ghabi; adil.el-ghabi@ced.uca.ma
}

Received 30 October 2020; Revised 18 December 2020; Accepted 30 December 2020; Published 5 March 2021

Academic Editor: Nawab Hussain

Copyright (C) 2021 Adil El-Ghabi et al. This is an open access article distributed under the Creative Commons Attribution License, which permits unrestricted use, distribution, and reproduction in any medium, provided the original work is properly cited.

\begin{abstract}
In this paper, we establish several random fixed point theorems for random operators satisfying some iterative condition w.r.t. a measure of noncompactness. We also discuss the case of monotone random operators in ordered Banach spaces. Our results extend several earlier works, including Itoh's random fixed point theorem. As an application, we discuss the existence of random solutions to a class of random first-order vector-valued ordinary differential equations with lack of compactness.
\end{abstract}

\section{Introduction}

Random fixed point theorems are stochastic generalizations of deterministic fixed point theorems. In recent years, random fixed point theorems have assumed importance due to their applications to random differential and integral equations (see for instance [1-9] and the references therein). Since Bharucha-Reid published his survey paper [10], numerous works of great importance have appeared and contributed strongly to the enrichment of the random fixed point theory. We quote, for instance, the contributions by Itoh [11], Reich [12], Rybinski [13], Sehgal and Singh [14], Sehgal and Waters [15], Papageorgiou [16], Tan and Yuan [17], Hussain et al. [18], and many others.

In 1979, Itoh [11] proved the random version of Sadovskii's well-known fixed point theorem [19]. Later on, Tan and Yuan [17] established an interesting result which serves as a bridge that links the random fixed point theory with the deterministic fixed point theory. Specifically, their result ensures the existence of a random fixed point for a continuous random operator under some compactness conditions provided that the corresponding deterministic fixed point problem is solvable. Recently, El-Ghabi and Taoudi [20] developed a new fixed point approach that combines the advantages of the strong topology with the advantages of the weak topology and which enables them to draw new and meaningful conclusions about random fixed points for a given random operator and to handle nonlinear random problems with lack of compactness.

In the present paper, we prove some new random fixed point theorems for (countably) convex-power condensing random operators in Banach spaces. Our results extend, generalize, and unify several known random fixed point results including the famous Itoh random fixed point theorem [11], Theorem 2.1. We should point out here that the concept of convex-power condensing operator was introduced in [21] as a generalization of the concept of condensing operator. We also prove some new random fixed point results for monotone (countably) convex-power condensing random operators in ordered Banach spaces. To illustrate our theoretical results, we investigate the solvability of a broad class of random first-order vector-valued ordinary differential equations. At this point, it is of significance to note that this class of random differential equations was already examined in [11] under some restriction on the constants used in the condition of measure of noncompactness and we have been successful in using our random fixed point results to remove this restriction (see Remark 34).

The paper is arranged as follows. In Section 2, we fix the notation and present some key tools that will be used to prove our main results. Section 3 is devoted to random fixed point theorems for (countably) convex-power condensing random operators and their corollaries. In Section 4, we prove some new random fixed point theorems for a class of 
monotone random operators in an ordered Banach space. Finally, in Section 5, we use some material from the previous sections to solve the random differential equation

$$
\frac{d}{d t} u(t, w)=f(t, u(t, w), w)
$$

where $t$ belongs to a bounded and closed interval in the real line $\mathbb{R}, \omega$ belongs to a set $\Omega$ endowed with a $\sigma$-algebra, and the functions $f, u$ have values in a Banach space $E$.

\section{Preliminaries}

Throughout this paper, $(\Omega, \Sigma)$ denotes a measurable space, where $\Omega$ is a nonempty set and $\Sigma$ is a $\sigma$-algebra of subsets of $\Omega$. Let $(E,\|\|$.$) be a Banach space, X$ be a nonempty subset of $E$ and $\mathcal{B}_{1}$ be the closed unit ball of $E$. Let $2^{X}, B(X)$, and $C D(X)$ denote, respectively, the collections of all nonempty subsets, bounded subsets, and closed subsets of $X$. In addition, let $K(X)$ and $W K(X)$ denote, respectively, the classes of compact and weakly compact subsets of $X$. We say that a mapping $T: X \longrightarrow E$ is hemicompact if each sequence $\left\{x_{n}\right\}_{n \in \mathbb{N}}$ in $X$ has a convergent subsequence whenever $\| x_{n}$ $-T\left(x_{n}\right) \| \longrightarrow 0$ as $n \longrightarrow \infty$. A mapping $F: \Omega \longrightarrow 2^{E}$ is said to be measurable if for every open subset $U$ of $E, F^{-1}(U)=$ $\{\omega \in \Omega: F(\omega) \cap U \neq \varnothing\} \in \Sigma$. It is important to stress here that, in the case where $F(\omega) \in K(E)$ for each $\omega \in \Omega, F$ is measurable if and only if $F^{-1}(C) \in \sum$ for each $C \in C D(E)$ (cf. [22], Theorem 3]). A mapping $T: \Omega \times X \longrightarrow E$ is called a random operator if $T(., x)$ is measurable for each $x \in X$. A mapping $\xi: \Omega \longrightarrow X$ is said to be:

(i) a deterministic fixed point of a random operator $T$ $: \Omega \times X \longrightarrow E$ if $T(\omega, \xi(\omega))=\xi(\omega)$ for every $\omega \in \Omega$,

(ii) a random fixed point of a random operator $T: \Omega \times$ $X \longrightarrow E$ if it is measurable and $T(\omega, \xi(\omega))=\xi(\omega)$ for every $\omega \in \Omega$,

Now, we present some basic facts regarding measures of (weak) noncompactness in Banach spaces, which we will be needed in the sequel. It is noteworthy that measures of noncompactness have played a substantial role in nonlinear functional analysis. They are very often used in the theory of functional equations, including ordinary differential equations, equations with partial derivatives, integral and integrodifferential equations, and optimal control theory. We also highlight that the interplay between fixed point theory and measures of noncompactness is very powerful and fruitful (see for instance $[19,23-29]$ and the references therein).

Definition 1 (see [30-32]). Let $E$ be a Banach space. A function $\psi: B(E) \longrightarrow[0,+\infty)$ is said to be a measure of (weak) noncompactness if it satisfies the following conditions:

(1) The family $\operatorname{ker}(\psi)=\{M \in B(E): \psi(M)=0\}$ is nonempty and $\operatorname{ker}(\psi)$ is contained in the set of relatively (weakly) compact sets of $E$.

(2) $M_{1} \subset M_{2} \Longrightarrow \psi\left(M_{1}\right) \leq \psi\left(M_{2}\right)$ for all $M_{1}, M_{2}$ in $B(E)$.
(3) $\psi(\overline{c o}(M))=\psi(M)$ for each $M \in B(E)$, where $\overline{c o}(M)$ denotes the closed convex hull of $M$

(4) $\psi\left(\lambda M_{1}+(1-\lambda) M_{2}\right) \leq \lambda \psi\left(M_{1}\right)+(1-\lambda) \psi\left(M_{2}\right)$ for all $\lambda \in[0 ; 1]$ and $M_{1}, M_{2} \in B(E)$.

(5) If $\left(M_{n}\right)_{n \geq 1}$ is a sequence of nonempty (weakly) closed subsets of $E$ with $M_{1}$ bounded and $M_{1} \supseteq M_{2} \supseteq \cdots \supseteq$ $M_{n} \supseteq \cdots$ such that $\lim _{n \rightarrow \infty} \psi\left(M_{n}\right)=0$, then $M_{\infty}:=\bigcap_{n=1}^{\infty}$ $M_{n}$ is nonempty.

The family ker $(\psi)$ is said to be the kernel of the measure of (weak) noncompactness $\psi$. Note that the intersection set $M_{\infty}$ belongs to $\operatorname{ker}(\psi)$ (see [33]). A measure of (weak) noncompactness $\psi$ is said to be

(6) Full if $\psi(M)=0$ if and only if $M$ is a relatively (weakly) compact set.

(7) Nonsingular if

$$
\psi(M \cup\{x\})=\psi(M)
$$

for all $M \in B(E)$ and $x \in E$

(8) Set additive or has the maximum property if

$$
\psi\left(M_{1} \cup M_{2}\right)=\max \left(\psi\left(M_{1}\right), \psi\left(M_{2}\right)\right) \text { for all } M_{1}, M_{2} \in B(E)
$$

(9) Homogeneous if

$$
\psi(\lambda M)=|\lambda| \psi(M) \text { for all } \lambda \in \mathbb{R} \text { and } M \in B(E)
$$

(10) Subadditive if

$$
\psi\left(M_{1}+M_{2}\right) \leq \psi\left(M_{1}\right)+\psi\left(M_{2}\right) \text { for all } M_{1}, M_{2} \in B(E)
$$

(11) Regular if $\psi$ is full, homogeneous, nonsingular, subadditive, and set additive.

The first important measure of weak noncompactness was defined by De Blasi [34] as follows:

$\beta(M)=\inf \left\{r>0\right.$ : there exists $W \in W K(E)$ with $\left.M \subset W+r \mathcal{B}_{1}\right\}$,

for each $M$ in $B(E)$. Notice that $\beta$ is regular (see [34]).

Also, the most important regular measures of noncompactness include the Kuratowski measure of noncompactness defined by

$$
\alpha(M)=\inf \left\{r>0: M \subseteq \bigcup_{i=1}^{n} M_{i}, \operatorname{diam}\left(M_{i}\right) \leq r, \forall i\right\}
$$


here $\operatorname{diam}\left(M_{i}\right)=\sup \left\{\|x-y\|: x, y \in M_{i}\right\}$. For more details, we refer the reader to the interesting book by Banaś and Goebel [30].

Now, we give a short note about (countably) convexpower condensing mappings with respect to a measure of (weak) noncompactness. Let $X$ be a nonempty closed convex subset of $E$ and $x_{0} \in X$. Let $T: X \longrightarrow X$ be a bounded mapping (i.e., $T$ takes bounded sets into bounded ones). Following $[33,35]$, for any $M \subset X$, we set

$$
\begin{gathered}
T^{\left(1, x_{0}\right)}(M)=T(M), \\
T^{\left(n, x_{0}\right)}(M)=T\left(\overline{c o}\left(T^{\left(n-1, x_{0}\right)}(M) \cup\left\{x_{0}\right\}\right)\right) \text { for, } n=2,3, .
\end{gathered}
$$

Definition 2. Let $E$ be a Banach space and $\psi$ be a measure of (weak) noncompactness on $E$. Let $X$ be a nonempty closed convex subset of $E$ and $T: X \longrightarrow X$ be a bounded mapping. We say that $T$ is (countably) $\psi$-convex-power condensing about $x_{0} \in X$ and $n_{0} \in \mathbb{N}$ if

$$
\psi\left(T^{\left(n_{0}, x_{0}\right)}(M)\right)<\psi(M)
$$

for any (countable) bounded subset $M$ of $X$ with $\psi(M)>0$.

It is customary to say "(countably) $\psi$-condensing" in place of " $\psi$-convex-power condensing about $x_{0}$ and 1 ." We also say that $T$ is (countably) $\psi$-convex-power condensing if there exist $x_{0} \in X$ and $n_{0} \in \mathbb{N}$ such that $T$ is (countably) $\psi$ -convex-power condensing about $x_{0}$ and $n_{0}$.

\section{Random Fixed Point Theorems for (Countably) Convex-Power Condensing Random Operators in Banach Spaces}

Throughout this section, $(\Omega, \Sigma)$ is a measurable space, $E$ is a Banach space and $X$ is a nonempty closed convex subset of $E$. Let $T: \Omega \times X \longrightarrow E$ be a random operator. We say that $T$ is continuous (resp., uniformly continuous, hemicompact) if for each $\omega \in \Omega, T(\omega$, .) is continuous (resp., uniformly continuous, hemicompact). If $T$ has values in $X$, we say that $T$ is (countably) $\psi$-convex-power condensing if $T(\omega,$.$) is$ (countably) $\psi$-convex-power condensing for every $\omega \in \Omega$. We say also that $T$ is $k($.)-lipschitzian (resp. a $k($.$) -contrac-$ tion) if there exists a mapping $k: \Omega \longrightarrow[0,+\infty)$ (resp.: $\Omega \longrightarrow[0,1)$ ) satisfying $\|T(\omega, x)-T(\omega, y)\| \leq k(\omega) \| x$ $-y \|$ for all $x, y \in X$ and all $\omega \in \Omega$.

The following result guarantees the existence of a random fixed point for a continuous random operator provided that the corresponding deterministic fixed point problem is solvable.

Theorem 3 (see [17], Theorem 2.3). Let $(\Omega, \Sigma)$ be a measurable space and $X$ be a nonempty separable complete subset of $a$ Banach space E. Suppose that T: $\Omega \times X \longrightarrow C D(E)$ is a continuous hemicompact random operator. Then, $T$ has a deterministic fixed point if and only if $T$ has a random fixed point.
Our main purpose in the immediate sequel is to prove some new random fixed point theorems for countably convex-power condensing mappings. Before proceeding further with the first main theorem, we obtain some auxiliary results.

Lemma 4. Let $E$ be a Banach space, $X$ be a closed convex subset of $E$ and $\psi$ be a regular measure of noncompactness on $E$. Let $T: X \longrightarrow X$ be a uniformly continuous countably $\psi$-convex-power condensing operator. If $T(X)$ is bounded, then $T$ is hemicompact.

Proof. Let $\Delta=\left\{x_{k}\right\}_{k \geq 0}$ be a sequence of elements of $X$ such that

$$
\left\|T\left(x_{k}\right)-x_{k}\right\| \longrightarrow 0 \text { as } k \longrightarrow \infty \text {. }
$$

We show that for each $n \in \mathbb{N}, \psi(\Delta) \leq \psi\left(T^{n}(\Delta)\right)$, where $T^{n}$ is the $n^{\text {th }}$ iterate of $T$. To see this, notice first that if $T$ is uniformly continuous, then so is $T^{n}$ for each integer $n$. Let $\varepsilon>0$ be fixed and take any integer $n$. Then, there exists $\delta>$ 0 such that for all $x, y \in X$ with $\|x-y\|<\delta$, we have $\| T^{n}(x)$ $-T^{n}(y) \|<\varepsilon$. By virtue of (10), there exists an integer $N$ such that for each $k \geq N$, we have $\left\|T\left(x_{k}\right)-x_{k}\right\|<\delta$, so that,

$$
\left\|T^{n+1}\left(x_{k}\right)-T^{n}\left(x_{k}\right)\right\|=\left\|T^{n}\left(T\left(x_{k}\right)\right)-T^{n}\left(x_{k}\right)\right\|<\varepsilon,
$$

whenever $k \geq N$. With this in mind, we can easily see that

$$
\left\{T^{n}\left(x_{k}\right)\right\}_{k \geq N} \subseteq\left\{T^{n+1}\left(x_{k}\right)\right\}_{k \geq N}+\varepsilon \mathscr{B}_{1} \subseteq T^{n+1}(\Delta)+\varepsilon \mathscr{B}_{1},
$$

where $\mathscr{B}_{1}$ is the closed unit ball of $E$. From the properties of the measure of noncompactness, it follows that

$$
\psi\left(\left\{T^{n}\left(x_{k}\right)\right\}_{k \geq N}\right) \leq \psi\left(T^{n+1}(\Delta)\right)+\varepsilon \psi\left(\mathscr{B}_{1}\right),
$$

which amounts to

$$
\psi\left(T^{n}(\Delta)\right)=\psi\left(\left\{T^{n}\left(x_{k}\right)\right\}_{k \geq N}\right) \leq \psi\left(T^{n+1}(\Delta)\right)+\varepsilon \psi\left(\mathscr{B}_{1}\right) .
$$

The arbitrariness of $\varepsilon$ yields $\psi\left(T^{n}(\Delta)\right) \leq \psi\left(T^{n+1}(\Delta)\right)$. Thus, $\left\{\psi\left(T^{n}(\Delta)\right)\right\}_{n \in \mathbb{N}}$ is an increasing sequence and therefore

$$
\psi(\Delta)=\psi\left(T^{0}(\Delta)\right) \leq \psi\left(T^{n}(\Delta)\right)
$$

for any integer $n \geq 0$. Let $a \in X$ and $n_{0} \in \mathbb{N}$ such that $T$ is countably $\psi$-convex-power condensing about $a$ and $n_{0}$. By mathematical induction, we can see that

$$
T^{n}(\Delta) \subseteq T^{(n, a)}(\Delta)
$$

for any integer $n \geq 1$. Going back to (15), we get

$$
\psi(\Delta) \leq T^{\left(n_{0}, a\right)}(\Delta)
$$


From our hypotheses, we know that $\psi(\Delta)=0$ and so $\Delta$ is relatively compact. Thus, the sequence $\left\{x_{k}\right\}_{k \geq 0}$ has a convergent subsequence and consequently $T$ is hemicompact.

Now, we are ready to state and prove the following deterministic fixed point theorem.

Theorem 5. Let $E$ be a Banach space and $X$ be a closed convex subset of E. Let $T: X \longrightarrow X$ be a continuous mapping such that $T(X)$ is bounded. Assume further that there are an integer $n_{0} \geq 1$ and a vector $x_{0} \in X$ such that for any countable subset $C \subset X$, we have

$$
\left[C \subseteq \overline{c o}\left(T^{\left(n_{0}, x_{0}\right)}(C) \cup\left\{x_{0}\right\}\right)\right] \Rightarrow[C \text { is relatively compact }]
$$

Then, T has a fixed point.

Proof. Consider the iterative sequence $\left(D_{n}\right)$ of sets given by

$$
D_{0}=\left\{x_{0}\right\}, D_{n}=\overline{c o}\left(T\left(D_{n-1}\right) \cup\left\{x_{0}\right\}\right) \text { for every } n \geq 1 \text {. }
$$

By mathematical induction, it is easy to show that $D_{n} \subseteq$ $D_{n+1}$ and $D_{n}$ is convex compact for each $n \geq 0$. Let $D=$ $\bigcup_{n \geq 0} D_{n}$. Plainly, $D$ is convex. Furthermore, observe that for each $n \in \mathbb{N}, D_{n}$ is separable (since it is compact), and hence, there exists a countable set $C_{n} \subseteq D_{n}$ such that $\bar{C}_{n}=D_{n}$. Let us consider the countable subset $C=\bigcup_{n \geq 0} C_{n}$ of $D$. It is readily apparent that $D=\bigcup_{n \geq 0} \bar{C}_{n} \subseteq{\overline{\bigcup_{n \geq 0} C_{n}}}=C$, and so $\bar{D}=\bar{C}$.

We claim that for each $k \geq 1$,

$$
C \subseteq \overline{c o}\left(T^{\left(k, x_{0}\right)}(C) \cup\left\{x_{0}\right\}\right)
$$

We proceed by induction. For the base case $k=1$, we can easily verify that

$$
\begin{aligned}
C \subset \bar{D} \subseteq \overline{c o}\left(T(D) \cup\left\{x_{0}\right\}\right) & =\overline{c o}\left(T(\bar{D}) \cup\left\{x_{0}\right\}\right) \\
& =\overline{c o}\left(T(\bar{C}) \cup\left\{x_{0}\right\}\right) \\
& =\overline{c o}\left(T(C) \cup\left\{x_{0}\right\}\right) \\
& =\overline{c o}\left(T^{\left(1, x_{0}\right)}(C) \cup\left\{x_{0}\right\}\right) .
\end{aligned}
$$

Let $k \geq 1$ be fixed and suppose that

$$
C \subseteq \overline{c o}\left(T^{\left(k, x_{0}\right)}(C) \cup\left\{x_{0}\right\}\right)
$$

Then,

$$
T(C) \subseteq T\left(\overline{c o}\left(T^{\left(k, x_{0}\right)}(C) \cup\left\{x_{0}\right\}\right)\right)=T^{\left(k+1, x_{0}\right)}(C),
$$

so that

$$
C \subset \overline{c o}\left(T(C) \cup\left\{x_{0}\right\}\right) \subseteq \overline{c o}\left(T^{\left(k+1, x_{0}\right)}(C) \cup\left\{x_{0}\right\}\right)
$$

This proves our claim. From our hypotheses, we deduce that $\bar{C}$ (and so $\bar{D}$ ) is compact. Furthermore, it is not difficult to see that

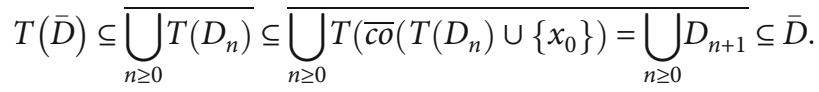

An appeal to Schauder's fixed point theorem yields a fixed point for $T$.

Remark 6. It is of significance to note that condition (18) is fulfilled whenever $T$ is (countably) $\psi$-convex-power condensing about $x_{0}$ and $n_{0}$.

After these preparations, we are now ready to state the main result of this section. This result ensures the existence of a random fixed point for a uniformly continuous random operator under general compactness conditions.

Theorem 7. Let E be a Banach space and $\psi$ be a regular measure of noncompactness on $E$. Let $X$ be a separable closed convex subset of $E$ and let $T: \Omega \times X \longrightarrow X$ be a uniformly continuous and countably $\psi$-convex-power condensing random operator. Assume that $T(\omega, X)$ is bounded for each $\omega \in$ $\Omega$, then, $T$ has a random fixed point.

Proof. Invoking Theorem 5, we infer that the random operator $T$ has a deterministic fixed point. In addition, since for each $\omega \in \Omega, T(\omega,):. X \longrightarrow X$ is a uniformly continuous countably $\psi$-convex-power condensing mapping and $T(\omega$, $X)$ is bounded, then by Lemma $4, T$ is hemicompact. The desired result follows from Theorem 3 .

Next, we turn our attention to the case when the boundary condition is of Rothe type. Before making a formal statement of our next random fixed point result, we need some auxiliary results. We first recall the following lemma.

Lemma 8 (see [15], Lemma 2). Let $f: \Omega \longrightarrow E$ and $l: \Omega$ $\longrightarrow[0,1]$ be measurable mappings. Then, for any $y \in E$, the mapping $h: \Omega \longrightarrow E$ defined by $h(\omega)=l(\omega) f(\omega)+(1-l(\omega)$ ) $y$ is measurable.

Recall that, if $U$ is a convex neighborhood of the origin in $E$, then the Minkowski functional $p$ of $U$ is defined on $E$ by

$$
p(x)=\inf \{\varepsilon>0: x \in \varepsilon U\} .
$$

It is well known [36], Lemma 5.12.1 that $p$ is continuous, subadditive, positively homogeneous, and satisfies

$$
\{x \in E: p(x)<1\} \subseteq U \subseteq\{x \in E: p(x) \leq 1\} \subseteq U .
$$

Note also that if $U$ has a nonempty interior, then $p$ is uniformly continuous.

Now, we state the following Browder-Fan type result [37], which is crucial for our purposes. 
Theorem 9. Let $X$ be a separable closed convex subset of a Banach space $E$ with Int $(X) \neq \varnothing$ and let $\psi$ be a regular measure of noncompactness on $E$. Let $x_{0} \in \operatorname{Int}(X), n_{0}: \Omega \longrightarrow \mathbb{N}$ be a mapping and $T: \Omega \times X \longrightarrow E$ be a uniformly continuous random operator. Assume that $T(\omega, X)$ is bounded and $T(\omega$ ,.) is (countably) $\psi$-convex-power condensing about $x_{0}$ and $n_{0}(\omega)$ for each $\omega \in \Omega$. Then, there exists a measurable mapping $\xi: \Omega \longrightarrow X$ satisfying

$$
p(T(\omega, \xi(\omega))-\xi(\omega))=\min \{p(T(\omega, \xi(\omega))-x): x \in X\},
$$

for each $\omega \in \Omega$, where $p$ is the Minkowski functional of (X$\left.x_{0}\right)$. Further, if $p\left(T(\omega, \xi(\omega))-x_{0}\right) \leq 1$ for some $\omega \in \Omega$, then $T(\omega, \xi(\omega))=\xi(\omega)$.

Proof. Let $p$ be the Minkowski functional of $\left(X-x_{0}\right)$ and define the mapping $l: \Omega \times X \longrightarrow[0,1]$ by

$$
l(\omega, x)=\left[\max \left\{1, p\left(T(\omega, x)-x_{0}\right)\right\}\right]^{-1}
$$

for each $(\omega, x) \in \Omega \times X$. Consider the mapping $g: \Omega \times X$ $\longrightarrow E$ defined by

$$
g(\omega, x)=l(\omega, x) T(\omega, x)+(1-l(\omega, x)) x_{0} .
$$

The reasoning in [15], Theorem 1 yields that $g$ is a continuous random operator such that for each $\omega \in \Omega, g(\omega, X)$ is bounded. We will show that $g$ satisfies all conditions of Theorem 7,. To this end, let $\omega \in \Omega$ be fixed. First notice that

$$
p\left(g(\omega, x)-x_{0}\right)=l(\omega, x) p\left(T(\omega, x)-x_{0}\right) \leq 1,
$$

for any $x \in X$, so that, $g(\Omega \times X) \subset X$. In addition, it is easy to check that

$$
|l(\omega, x)-l(\omega, y)| \leq\left|p\left(T(\omega, x)-x_{0}\right)-p\left(T(\omega, y)-x_{0}\right)\right|,
$$

for all $x, y \in E$. Since $p$ and $T(\omega,$.$) are uniformly continuous,$ so is $l(\omega,$.$) . Furthermore, for all x, y \in X$, we obtain

$$
\begin{aligned}
\|g(\omega, x)-g(\omega, y)\|= & \| l(\omega, x)\left(T(\omega, x)-x_{0}\right) \\
& -l(\omega, y)\left(T(\omega, y)-x_{0}\right) \| \\
= & \| l(\omega, x)(T(\omega, x)-T(\omega, y))+(l(\omega, x) \\
& -l(\omega, y))\left(T(\omega, y)-x_{0}\right) \| \\
\leq & l(\omega, x)\|T(\omega, x)-T(\omega, y)\|+\mid l(\omega, x) \\
& -l(\omega, y) \mid\left(\|T(\omega, y)\|+\left\|x_{0}\right\|\right) \\
\leq & \|T(\omega, x)-T(\omega, y)\|+\mid l(\omega, x) \\
& -l(\omega, y) \mid\left(\|T(\omega, .)\|_{\infty}+\left\|x_{0}\right\|\right) .
\end{aligned}
$$

It follows that $g(\omega,$.$) is uniformly continuous. Next, we$ will illustrate that $g(\omega,$.$) is countably \psi$-convex-power condensing about $x_{0}$ and $n_{0}(\omega)$. To see this, take any bounded countable subset $M$ of $X$ with $\psi(M)>0$. We claim that $g^{\left(k, x_{0}\right)}(\omega, M) \subseteq \overline{c o}\left(T^{\left(k, x_{0}\right)}(\omega, M) \cup\left\{x_{0}\right\}\right)$ for each $k \geq 1$. Indeed, for $k=1$, by (30), we have $g(\omega, M) \subset \overline{c o}(T(\omega, M) \cup$ $\left.\left\{x_{0}\right\}\right)$, so that

$$
g^{\left(1, x_{0}\right)}(\omega, M) \subset \overline{c o}\left(T^{\left(1, x_{0}\right)}(\omega, M) \cup\left\{x_{0}\right\}\right)
$$

Let $k \geq 1$ be fixed and assume that $g^{\left(k, x_{0}\right)}(\omega, M) \subseteq \overline{c o}($ $\left.T^{\left(k, x_{0}\right)}(\omega, M) \cup\left\{x_{0}\right\}\right)$.

Then,

$$
\begin{aligned}
g^{\left(k+1, x_{0}\right)}(\omega, M) & =g\left(\overline{c o}\left(g^{\left(k, x_{0}\right)}(\omega, M) \cup\left\{x_{0}\right\}\right)\right) \\
& \subseteq \overline{c o}\left(T\left(\omega, \overline{c o}\left(T^{\left(k, x_{0}\right)}(\omega, M) \cup\left\{x_{0}\right\}\right)\right) \cup\left\{x_{0}\right\}\right) \\
& =\overline{c o}\left(T^{\left(k+1, x_{0}\right)}(\omega, M) \cup\left\{x_{0}\right\}\right) .
\end{aligned}
$$

This proves our claim. Thus,

$$
\begin{aligned}
\psi\left(g^{\left(n_{0}(\omega), x_{0}\right)}(\omega, M)\right) & \leq \psi\left(\overline{c o}\left(T^{\left(n_{0}(\omega), x_{0}\right)}(\omega, M) \cup\left\{x_{0}\right\}\right)\right) \\
& =\psi\left(T^{\left(n_{0}(\omega), x_{0}\right)}(\omega, M)\right)<\psi(M) .
\end{aligned}
$$

Consequently, $g(\omega,$.$) is countably \psi$-convex-power condensing about $x_{0}$ and $n_{0}(\omega)$. Now, by applying Theorem 7 , we infer that there is a measurable mapping $\xi: \Omega \longrightarrow X$ such that for each $\omega \in \Omega$, we have $g(\omega, \xi(\omega))=\xi(\omega)$, that is,

$$
l(\omega, \xi(\omega)) T(\omega, \xi(\omega))+(1-l(\omega, \xi(\omega))) x_{0}=\xi(\omega) .
$$

Now, for any $\omega \in \Omega$, either (a) $p\left(T(\omega, \xi(\omega))-x_{0}\right) \leq 1$ or (b) $p\left(T(\omega, \xi(\omega))-x_{0}\right)>1$. In case of (a), it follows by (29) that $l(\omega, \xi(\omega))=1$ and hence by $(37), T(\omega, \xi(\omega))=\xi(\omega)$. If (b) holds, then by (29), we have

$$
l(\omega, \xi(\omega)) p\left(T(\omega, \xi(\omega))-x_{0}\right)=1,
$$

and since for every $x \in X, p\left(x-x_{0}\right) \leq 1$, it follows by (37) that for every $x \in X$, we have

$$
T(\omega, \xi(\omega))-\xi(\omega))=(1-l(\omega, \xi(\omega)))\left(T(\omega, \xi(\omega))-x_{0}\right)
$$

Then,

$p(T(\omega, \xi(\omega))-\xi(\omega)) \leq\left(p\left(T(\omega, \xi(\omega))-x_{0}\right)-1\right) p\left(\xi(\omega)-x_{0}\right)$.

Since $\xi(\omega) \in X$, the last inequality implies that

$$
\begin{aligned}
p(T(\omega, \xi(\omega))-\xi(\omega)) & \leq p\left(T(\omega, \xi(\omega))-x_{0}\right)-1 \\
& \leq p(T(\omega, \xi(\omega))-x),
\end{aligned}
$$


and

$$
p(T(\omega, \xi(\omega))-\xi(\omega))=\min \{p(T(\omega, \xi(\omega))-x): x \in X\} .
$$

Thus, (a) and (b) provide the desired conclusion.

With these preliminaries, we can proceed to the following interesting random fixed point theorem under Rothe-type boundary conditions.

Theorem 5. Let $X$ be a separable closed convex subset of a Banach space $E$ with Int $(X) \neq \varnothing$ and let $\psi$ be a regular measure of noncompactness on $E$. Let $n_{0}: \Omega \longrightarrow \mathbb{N}$ be a mapping and $T: \Omega \times X \longrightarrow E$ be a uniformly continuous random operator. Let $x_{0} \in \operatorname{Int}(X)$ such that for each $\omega \in \Omega, T(\omega, X)$ is bounded and $T(\omega,$.$) is countably \psi$-convex-power condensing about $x_{0}$ and $n_{0}(\omega)$. In addition, assume that $T(\Omega$ $\times \partial X) \subseteq X$ (Rothe boundary condition). Then, $T$ has a random fixed point.

Proof. Let $p$ be the Minkowski functional of $\left(X-x_{0}\right)$. By Theorem 9 , there is a measurable mapping $\xi: \Omega \longrightarrow X$ satisfying (28). To prove that $\xi$ is a random fixed point of $T$, it suffices to show that $p\left(T(\omega, \xi(\omega))-x_{0}\right) \leq 1$ for each $\omega \in \Omega$. Suppose that for some $\omega \in \Omega$, we have $p\left(T(\omega, \xi(\omega))-x_{0}\right)>1$, then $T$ $(\omega, \xi(\omega)) \notin X$. This implies that $\xi(\omega) \in \operatorname{Int}(X)$. Consequently, there is $0<\eta<1$ such that $z=\eta \xi(\omega)+(1-\eta) T(\omega, \xi(\omega)) \epsilon$ $\partial X$. Hence, by (28), we have

$p(T(\omega, \xi(\omega))-\xi(\omega)) \leq p(T(\omega, \xi(\omega))-z)=\eta p(T(\omega, \xi(\omega))-\xi(\omega))$. that

Thus, $p(T(\omega, \xi(\omega))-\xi(\omega))=0$. This obviously yields

$$
p\left(T(\omega, \xi(\omega))-x_{0}\right) \leq p\left(\xi(\omega)-x_{0}\right) \leq 1,
$$

which contradicts the assumption.

Our next concern will be the existence of random fixed points for the sum of two random operators. We should mention here that the need for such random fixed point theorems arose out of the study of random differential equations. Specifically, the inversion of a random differential operator may yield the sum of a contraction and an operator satisfying some compactness conditions. Before, to state our next random fixed point result, we need to recall some basic facts.

Lemma 3 (see [20], Lemma 2.11). Let $E$ be a separable Banach space and $S: \Omega \times E \longrightarrow E$ be a $k($.$) -contraction ran-$ dom operator. Then,

(i) for each $x \in E$ there is a unique measurable function $\tau_{x}: \Omega \longrightarrow E$ such that for each $\omega \in \Omega: \tau_{x}(\omega)=S(\omega$, $\left.\tau_{x}(\omega)\right)+x$

(ii) the mapping $\tau: \Omega \times E \longrightarrow E$ defined by $\tau(\omega, x)=\tau_{x}($ $\omega)$ is a Lipschitzian random operator
Lemma 4 (see [20], Lemma 2.12). Let $X$ be a closed and convex subset of a separable Banach space $E, T: \Omega \times X \longrightarrow E$ be a continuous random operator, and $x: \Omega \longrightarrow X$ be measurable. Then, the mapping $\omega \longrightarrow T(\omega, x(\omega))$ is measurable.

Now, we are in a position to state the following random fixed point theorem for the sum of two random operators. For convenient purposes, we list some necessary definitions below for completeness. Let $X$ be a nonempty bounded closed convex subset of a Banach space $E$ and $T: \Omega \times X$ $\longrightarrow E$ and $S: \Omega \times E \longrightarrow E$ be two random operators. Following [24,32,38], we set for any $\omega \in \Omega$ and for any subset $M$ of $X$ :

$$
\begin{aligned}
\mathcal{F}^{\left(1, x_{0}\right)}(\omega, T, S, M)= & \mathcal{F}(\omega, T, S, M)=\{x \in X: x=S(\omega, x) \\
& +T(\omega, y), \text { for some } y \in M\}
\end{aligned}
$$

and

$$
\begin{gathered}
\mathcal{F}^{\left(n, x_{0}\right)}(\omega, T, S, M)=\mathcal{F}\left(\omega, T, S, \overline{c o}\left(\mathcal{F}^{\left(n-1, x_{0}\right)}(\omega, T, S, M) \cup\left\{x_{0}\right\}\right)\right), \\
n=2,3, \ldots
\end{gathered}
$$

In the case when $S=0$, we have

$$
\mathcal{F}^{\left(1, x_{0}\right)}(\omega, T, 0, M)=T^{\left(1, x_{0}\right)}(\omega, M)=T(\omega, M)
$$

and

$$
\begin{aligned}
\mathcal{F}^{\left(n, x_{0}\right)}(\omega, T, 0, M) & =T\left(\omega, \bar{c} \bar{o}\left(\mathcal{F}^{\left(n-1, x_{0}\right)}(\omega, T, 0, M) \cup\left\{x_{0}\right\}\right)\right) \\
& =T^{\left(n, x_{0}\right)}(\omega, M) .
\end{aligned}
$$

Theorem 6. Let $X$ be a nonempty bounded closed convex subset of a separable Banach space $E$ and $\psi$ be a regular measure of noncompactness on $E$. Let $T: \Omega \times X \longrightarrow E$ and $S: \Omega \times E$ $\longrightarrow E$ be random operators satisfying the following conditions:

(i) $T$ is uniformly continuous

(ii) there are mappings $n_{0}: \Omega \longrightarrow N$ and $x_{0}: \Omega \longrightarrow X$ such that

$$
\psi\left(\mathscr{F}^{\left(n_{0}(\omega), x_{0}(\omega)\right)}(\omega, T, S, M)\right)<\psi M,
$$

for every $\omega \in \Omega$ and for any countable subset $M$ of $X$ with $\psi(M)>0$,

(iii) $S$ is a $k($.$) -contraction$

(iv) for each $\omega \in \Omega,[x=T(\omega, y)+S(\omega, x)$ and $y \in X] \Longrightarrow$ $x \in X$

Then, $T+S$ has a random fixed point. 
Proof. Consider the mapping $f: \Omega \times X \longrightarrow E$ defined by

$$
f(\omega, x)=\tau(\omega, T(\omega, x)),
$$

where $\tau$ is as described in Lemma 3. Referring to Lemma 4, we see that every $x \in X, f(., x)=\tau(., T(., x))$ is measurable. Thus, $f$ is a random operator. In addition, the fact that $T$ is uniformly continuous and $\tau$ is Lipschitzian imply that $f$ is uniformly continuous. Now, let $\omega \in \Omega$ be fixed. We claim that $f(\omega, X) \subseteq X$. Indeed, by virtue of Lemma 3 (i), we infer that for any $x \in X$ we have

$$
\tau(\omega, T(\omega, x))=S(\omega, \tau(\omega, T(\omega, x)))+T(\omega, x),
$$

so that,

$$
f(\omega, x)=S(\omega, f(\omega, x))+T(\omega, x) .
$$
claim.

Hence, by (iv), we have $f(\omega, x) \in X$. This proves our Furthermore, for any subset $M$ of $X$, by (52), we obtain

$$
\begin{aligned}
f^{\left(1, x_{0}(\omega)\right)}(\omega, M)= & f(\omega, M) \\
= & \{x \in X: x=f(\omega, y) \text { for some } y \in M\} \\
\subseteq & \{x \in X: x=S(\omega, x) \\
& +T(\omega, y) \text { for some } y \in M\} \\
= & \mathscr{F}^{\left(1, x_{0}(\omega)\right)}(\omega, T, S, M) .
\end{aligned}
$$

Let $n \geq 1$, suppose that

$$
f^{\left(n, x_{0}(\omega)\right)}(\omega, M) \subseteq \mathcal{F}^{\left(n, x_{0}(\omega)\right)}(\omega, T, S, M) .
$$

We have

$$
\begin{aligned}
f^{\left(n+1, x_{0}(\omega)\right)}(\omega, M) & =f\left(\omega, \bar{c} \bar{o}\left(f^{\left(n, x_{0}(\omega)\right)}(\omega, M) \cup\left\{x_{0}(\omega)\right\}\right)\right) \\
& \subseteq f\left(\omega, \bar{c} \bar{o}\left(\mathscr{F}^{\left(n, x_{0}(\omega)\right)}(\omega, T, S, M) \cup\left\{x_{0}(\omega)\right\}\right)\right) \\
& \subseteq \mathscr{F}\left(\omega, T, S, \bar{c} \bar{o}\left(\mathscr{F}^{\left(n, x_{0}(\omega)\right)}(\omega, T, S, M) \cup\left\{x_{0}(\omega)\right\}\right)\right) \\
& =\mathscr{F}^{\left(n+1, x_{0}(\omega)\right)}(\omega, T, S, M) .
\end{aligned}
$$

Hence, for each $n \geq 1$, we have

$$
f^{\left(n, x_{0}(\omega)\right)}(\omega, M) \subseteq \mathscr{F}^{\left(n, x_{0}(\omega)\right)}(\omega, T, S, M) .
$$

Thus, by (ii), we have

$$
\psi\left(f^{\left(n_{0}(\omega), x_{0}(\omega)\right)}(\omega, M)\right)<\psi(M),
$$

for any countable subset $M$ of $X$ with $\psi(M)>0$. As a result, $f(\omega,$.$) is countably \psi$-convex-power condensing about $x_{0}($ $\omega)$ and $n_{0}(\omega)$. Consequently, $f$ is countably $\psi$-convex-power condensing. Invoking Theorem 7 , we see that $f$ has a random fixed point $\xi: \Omega \longrightarrow X$ which is in turn a random fixed point of $T+S$.

Corollary 10. Let $X$ be a nonempty bounded closed convex subset of a separable Banach space $E$ and $\psi$ be a measure of noncompactness on $E$. Let $T: \Omega \times X \longrightarrow E$ and $S: \Omega \times E$ $\longrightarrow E$ be random operators satisfying the following conditions:

(i) $T$ is uniformly continuous

(ii) there are mappings $n_{0}: \Omega \longrightarrow \mathbb{N}, x_{0}: \Omega \longrightarrow X$ and $\lambda: \Omega \longrightarrow[0,1)$ such that

$$
\psi\left(\mathscr{F}^{\left(n_{0}(\omega), x_{0}(\omega)\right)}(\omega, T, S, M)\right) \leq \lambda(\omega) \psi(M)
$$

for every $\omega \in \Omega$ and for any countable subset $M$ of $X$

(iii) $S$ is a $k($.$) -contraction$

(iv) for each $\omega \in \Omega,[x=T(\omega, y)+S(\omega, x)$ and $y \in X] \Longrightarrow$ $x \in X$. Then, $T+S$ has a random fixed point

\section{Random Fixed Point Theorems for Monotone Random Operators}

In this section, we prove some random fixed point theorems for monotone random operators in ordered real Banach spaces. We combine the advantages of the strong topology (continuity of random operators with respect to the strong topology) with the advantages of the weak topology (the random operators will satisfy some compactness conditions relative to the weak topology) to draw new conclusions about random fixed points for a given monotone random operator. Our results are random versions of the results in [39].

We start this section by recalling some definitions and auxiliary results which will be used further on. Throughout this section, $(\Omega, \Sigma)$ is a measurable space, $E$ is a real Banach space, and $X$ is a nonempty subset of $E$.

Definition 11. A subset $P$ of $E$ is called an order cone if it satisfies the following conditions:

(i) $P$ is closed, nonempty and $P \neq\{0\}$,

(ii) $\forall a, b \in \mathbb{R}^{+}, \forall x, y \in P: a x+b y \in P$

(iii) $\mid x \in P$ and $-x \in P \mid \Rightarrow x=0$.

An order cone permits to define a partial order in $E$ by

$$
x \leq y \Leftrightarrow y-x \in P .
$$

Conversely, let $E$ be a real Banach space with a partial order compatible with the algebraic operations in $E$, that is,

$$
\begin{gathered}
x \geq 0 \text { and } \lambda \in \mathbb{R}^{+} \text {implies } \lambda x \geq 0, \\
x_{1} \leq y_{1} \text { and } x_{2} \leq y_{2} \text { implies } x_{1}+x_{2} \leq y_{1}+y_{2} .
\end{gathered}
$$


The positive cone of $E$ is defined by

$$
E^{+}=\{x \in E: 0 \leq x\}
$$
by

Let $x, y \in E$ with $x \leq y$, the order interval $[x, y]$ is defined

$$
[x, y]=\{z \in E: x \leq z \leq y\}=(x+P) \cap(y-P) .
$$

Definition 12.

(i) A subset $X \subset E$ is said order bounded if there exist $u$ ,$v \in E$ such that $X \subset[u, v]$

(ii) The order cone is called normal if and only if there is a number $C>0$ such that

$$
\forall x, y \in E:(0 \leq x \leq y) \Rightarrow(\|x\| \leq C\|y\|)
$$

The least positive number $C$ (if it exists) satisfying (63) is called a normal constant.

\section{Remarks 13.}

(1) If $P$ is normal, then every order interval is norm bounded.

(2) Let $K$ be a Hausdorff space and $E$ be an ordered Banach space with normal cone $P$. We denote by $\mathscr{C}$ $(K, E)$ the Banach space of all continuous $E$-valued functions on $K$ equipped with the usual maximum norm. Plainly, $\mathscr{C}(K, E)$ is an ordered Banach space whose positive cone is given by

$$
\mathscr{C}^{+}(K, E)=\left\{f \in \mathscr{C}(K, E): f(x) \in E^{+}, \forall x \in K\right\},
$$

and it is normal

Definition 14. Let $X$ be a subset of $E$ and $T: X \longrightarrow E$ be an operator.

(i) The operator $T$ is said to be increasing if

$$
\forall x, y \in X: x \leq y \Rightarrow T x \leq T y
$$

(ii) The operator $T$ is said to be decreasing if

$$
\forall x, y \in X: x \leq y \Rightarrow T y \leq T x
$$

Definition 15. Let $E$ be an ordered real Banach space with a normal order cone $P$. A sequence $\left\{x_{n}\right\}$ is said to be totally ordered if for all $m, n \in \mathbb{N}: x_{m} \leq x_{n}$ or $x_{n} \leq x_{m}$.

The following lemmas are quite useful below.
Lemma 16 (see [27], Lemma 2.1). Let $E$ be an ordered real Banach space with a normal order cone P. Suppose that $\left\{x_{n}\right.$ \} is a monotone sequence which has a subsequence $\left\{x_{n k}\right\}$ converging weakly to $x_{\infty}$. Then, $\left\{x_{n}\right\}$ converges strongly to $x_{\infty}$. Moreover, if $\left\{x_{n}\right\}$ is an increasing sequence then $x_{n} \leq x_{\infty}$ for each $n \geq 1$; if $\left\{x_{n}\right\}$ is a decreasing sequence then $x_{\infty} \leq x_{n}$ for each $n \geq 1$.

Lemma 17 (see [39], Lemma 1.8). Let $E$ be an ordered real Banach space with a normal order cone P. Suppose that $\left\{x_{n}\right.$ \} is a totally ordered sequence which is contained in a relatively weakly compact set, then it converges strongly in $E$.

Let $u_{0}, v_{0}: \Omega \longrightarrow X$ be two measurable mappings. By $u_{0} \leq v_{0}$ (resp., $u_{0}<v_{0}$ ) on $\Omega$, we mean $u_{0}(\omega) \leq v_{0}(\omega)$ (resp., $\left.u_{0}(\omega)<v_{0}(\omega)\right)$ for every $\omega \in \Omega$. If $u_{0} \leq v_{0}$, the sector $\left[u_{0}, v_{0}\right]$ defined by

$$
\left[u_{0}, v_{0}\right]=\left\{x: \Omega \rightarrow X: u_{0} \leq x \leq v_{0}\right\}
$$

is called a random interval in $X$.

Let $T: \Omega \times E \longrightarrow E$ be a random operator. For all $n \in N$, $\omega \in \Omega$, and $x \in X$, we denote by $T^{n}(\omega, x)$ the value at $x$ of the $n^{\text {th }}$ iterate of the mapping $T(\omega,$.$) . We say that a random$ operator $T: \Omega \times X \longrightarrow E$ satisfies the condition $\left(P\left(n_{0}\right)\right)$ on $\left[u_{0}, v_{0}\right]$ if there is a mapping $n_{0}: \Omega \rightarrow \mathbb{N}$ such that for each $\omega \in \Omega$,

$$
\left\{\begin{array}{l}
\text { if } V=\left\{x_{n}\right\} \text { is a monotone sequence of }\left[u_{0}(\omega), v_{0}(\omega)\right] \\
\text { such that } V=F \cup T^{n_{0}(\omega)}(\omega, V) \text { with } F \text { is a finite set of } \\
\text { cardinal } \mathrm{n}_{0}(\omega) \text {, then } V \text { is relatively weakly compact. }
\end{array}\right.
$$

We say that a random operator $T: \Omega \times X \longrightarrow E$ satisfies the condition $(P(1))$ on $\left[u_{0}, v_{0}\right]$ if $T$ satisfies $\left(P\left(n_{0}\right)\right)$ with $n_{0}(\omega)=1$ for every $\omega \in \Omega$.

Definition 18. Let $\left[u_{0}, v_{0}\right]$ be a random interval in $X$ with $u_{0}$ $\leq v_{0}$. A random operator $T: \Omega \times X \longrightarrow E$ is called increasing (resp., decreasing) on $\left[u_{0}, v_{0}\right]$ if for each $\omega \in \Omega, T(\omega,$. is increasing (resp., decreasing) on $\left[u_{0}(\omega), v_{0}(\omega)\right]$.

Theorem 19. Let $E$ be an ordered separable real Banach space with a normal cone $P$ and $T: \Omega \times E \longrightarrow E$ be a continuous random operator. Let $\left[u_{0}, v_{0}\right]$ be a random interval in $E$ such that $T$ is increasing on $\left[u_{0}, v_{0}\right]$ and satisfies the condition $(P($ $\left.n_{0}\right)$ ). Assume that

$$
u_{0}(.) \leq T\left(., u_{0}(.)\right), T\left(., v_{0}(.)\right) \leq v_{0}(.)
$$

Then, $T$ has a random fixed point $\xi$ in $\left[u_{0}, v_{0}\right]$ which can be obtained by monotone iterative procedure starting from $u_{0}$ or from $v_{0}$.

Proof. Consider the iterate sequence of mappings $u_{n}: \Omega$ $\longrightarrow E$ defined by $u_{n}(\omega)=T\left(\omega, u_{n-1}(\omega)\right)$, for all $\omega \in \Omega$ and 
all $n \in N$. Let $\omega \in \Omega$ be fixed. We can show by induction that

$$
u_{0}(\omega) \leq u_{1}(\omega) \leq u_{2}(\omega) \leq \cdots \leq u_{n}(\omega) \cdots \leq v_{0}(\omega)
$$

Let us set $S(\omega)=\left\{u_{n}(\omega): n \in \mathbb{N}\right\}$. Then,

$$
S(\omega)=T^{n_{0}(\omega)}(\omega, S(\omega)) \cup F(\omega)
$$

with $F(\omega)=\left\{u_{0}(\omega), u_{1}(\omega), \cdots, u_{n_{0}(\omega)-1}(\omega)\right\}$ is a finite set of cardinal $n_{0}(\omega)$. Therefore, it follows from our hypotheses that $S(\omega)$ is relatively weakly compact. Referring to Lemma 17 , we see that the sequence $\left\{u_{n}(\omega)\right\}$ converges strongly to some $\xi(\omega)$ in $\left[u_{0}(\omega), v_{0}(\omega)\right]$. The continuity of $T(\omega,$.$) yields$ that $T(\omega, \xi(\omega))=\xi(\omega)$. Now, by using Lemma 4 , we can show (via induction) that $u_{n}$ is measurable for every $n \in \mathbb{N}$. The use of [8], Theorem 1.6 yields that $\xi: \Omega \longrightarrow E$ is measurable; and therefore, $\xi$ is a random fixed point of $T$. Similarly, we can show that $T$ has random fixed point obtained by monotone iterative procedure starting from $v_{0}$.

As a convenient specialization of Theorem 19, we state the following result.

Corollary 20. Let $E$ be an ordered separable real Banach space with a normal cone $P$ and $T: \Omega \times E \longrightarrow E$ be a continuous random operator. Let $\left[u_{0}, v_{0}\right]$ be a random interval in $E$ such that $T$ is increasing on $\left[u_{0}, v_{0}\right]$ and satisfies the condition $(\mathcal{P}$ (1)). Assume that

$$
u_{0}(.) \leq T\left(., u_{0}(.)\right), T\left(., v_{0}(.)\right) \leq v_{0}(.) \text {. }
$$

Then, $T$ has a random fixed point $\xi$ in $\left[u_{0}, v_{0}\right]$ which can be obtained by monotone iterative procedure starting from $u_{0}$ or from $v_{0}$.

Proof. Apply Theorem 19 with $n 0(\omega)=1$ for every $\omega \in \Omega$.

Another consequence of Theorem 19 is the following.

Corollary 21. Let $E$ be an ordered separable real Banach space with a normal cone $P$ and $\psi$ be a nonsingular measure of weak noncompactness on $E$. Let $T: \Omega \times E \longrightarrow E$ be a continuous random operator and let $\left[u_{0}, v_{0}\right]$ be a random interval in $E$ such that $T$ is increasing on $\left[u_{0}, v_{0}\right]$ and

$$
u_{0}(.) \leq T\left(., u_{0}(.)\right), T\left(., v_{0}(.)\right) \leq v_{0}(.) \text {. }
$$

In addition, if there is a mapping $n_{0}: \Omega \longrightarrow N$, such that for each $\omega \in \Omega$ and any monotone sequence $\Gamma=\left\{x_{n}\right\}$ in $\left[u_{0}(\right.$ $\left.\omega), v_{0}(\omega)\right]$ with $\psi(\Gamma)>0$, we have

$$
\psi\left(T^{n_{0}(\omega)}(\omega, \Gamma)\right)<\psi(\Gamma) .
$$

Then, $T$ has a random fixed point $\xi$ in $\left[u_{0}, v_{0}\right]$ which can be obtained by monotone iterative procedure starting from $u_{0}$ or from $v_{0}$.

Proof. Let $\omega \in \Omega$ be fixed and let $V=\left\{x_{n}\right\}$ be a monotone sequence of $\left[u_{0}(\omega), v_{0}(\omega)\right]$ such that $V=F \cup T^{n 0(\omega)}(\omega, V)$ with $F$ is a finite set of cardinal $n_{0}(\omega)$. By (74), $V$ is relatively weakly compact. Thus, $T(\omega,$.$) satisfies the condition \left(\mathscr{P}\left(n_{0}\right)\right.$ ) on $\left[u_{0}(\omega), v_{0}(\omega)\right]$. The arbitrariness of $\omega$ yields that $T$ satisfies the condition $\left(P\left(n_{0}\right)\right)$ on the random interval $\left[u_{0}, v_{0}\right]$. The desired result follows from Theorem 19.

Corollary 22. Let E be an ordered separable real Banach space with a normal cone $P$ and $\psi$ be a measure of weak noncompactness on $E$.

Let $T: \Omega \times X \longrightarrow E$ be a continuous random operator and let $\left[u_{0}, v_{0}\right]$ be a random interval in $E$ such that $T$ is increasing on $\left[u_{0}, v_{0}\right]$ and

$$
u_{0}(.) \leq T\left(., u_{0}(.)\right), T\left(., v_{0}(.)\right) \leq v_{0}(.)
$$

In addition, assume that there is a mapping $n_{0}: \Omega \longrightarrow N$ such that for each $\omega \in \Omega$,

$$
T^{n_{0}(\omega)}\left(\omega,\left[u_{0}(\omega), v_{0}(\omega)\right]\right):=\left\{T^{n_{0}(\omega)}(\omega, x(\omega)): x \in\left[u_{0}, v_{0}\right]\right\},
$$

is relatively weakly compact. Then, $T$ has a random fixed point $\xi$ in $\left[u_{0}, v_{0}\right]$ which can be obtained by monotone iterative procedure starting from $u_{0}$ or from $v_{0}$.

\section{Applications to Random Differential Equations}

Throughout this section, $(\Omega, \Sigma)$ denotes a measurable space and $E$ is a separable real Banach space. Let $r, T>0, x_{0} \in E$, $\mathscr{B}=\left\{x \in E:\left\|x-x_{0}\right\| \leq r\right\}$ and $I=[0, T]$. Denote by $C(I ; E)$ the Banach space of all continuous mappings $u: I \longrightarrow E$ equipped with the supremum norm $\|u\|_{\infty}=\sup \{\|u(t)\|: t$ $\in I\}$ and by $C^{1}(I ; E)$ the Banach space of all continuously differentiable mappings from $I$ to $E$. Let $\alpha$ (resp., $\alpha_{C}$ ) be the Kuratowski measure of noncompactness on $E(\operatorname{resp} . \mathscr{C}(I ; E))$.

Definition 23. A mapping $u: I \times \Omega \longrightarrow E$ is said to satisfy the following conditions:

(1) $(C, \Omega)$ if for each $\omega \in \Omega, u(., \omega)$ is continuous and for each $t \in I, u(t$, .) is measurable

(2) $\left(C^{1}, \Omega\right)$ if $u(., \omega) \in C^{1}(I ; E)$ for every $\omega \in \Omega$ and $u(t$, .) is measurable for every $t \in I$

If $u$ satisfies condition $(C, \Omega)$, then $u$ is considered a mapping of $\Omega$ into $\mathscr{C}(I, E)$. The following result, due to Itoh, discusses the measurability of $u$.

Proposition 24 (see [11], Proposition 4.2). u satisfies condition $(C, \Omega)$ if and only if $u$ is measurable as a mapping of $\Omega$ into $C(I ; E)$. 
Now, we consider the initial random differential equation

$$
\left\{\begin{array}{l}
\frac{d}{d t} u(t, \omega)=f(t, u(t, \omega), \omega), \quad \text { for }(t, \omega) \in I \times \Omega, \\
u(0, \omega)=\eta(\omega)
\end{array}\right.
$$

where $f(. ., \omega) \in C(I \times E, E)$ and the function $\eta: \Omega \longrightarrow E$ is measurable. Our main purpose in the immediate sequel is to show the existence of a random solution to Eq. (77). Before doing so, it is appropriate to clarify the definition of solution we will consider.

Definition 25. A mapping $u: I \times \Omega \longrightarrow E$ is said to be a random solution of (77) if $u$ satisfies conditions $\left(C^{1}, \Omega\right)$ and (77).

For convenience of later reference, we list some necessary results below for completeness.

Lemma 26 (see [30]). Let $E$ be a Banach space and $C([0, T]$ ;E) be the space of continuous functions defined on $[0, T]$ with values in E. If $H \subset C([0, T] ; E)$ is bounded, then $\alpha(H(t)) \leq$ $\alpha_{C}(H)$ for any $t \in[0, T]$, where $H(t)=\{u(t): u \in H\}$. Furthermore, if $H$ is equicontinuous, then $t \longmapsto \alpha(H(t))$ is continuous on $[0, T]$,

$$
\begin{gathered}
\alpha_{C}(H)=\sup \{\alpha(H(t)): t \in[0, T]\}, \\
\alpha\left(\int_{0}^{t} H(s) d s\right) \leq \int_{0}^{t} \alpha(H(s)) d s,
\end{gathered}
$$

for all $t \in[0, T]$, where $\int_{0}^{t} H(s) d s=\left\{\int_{0}^{t} x(s) d s: x \in H\right\}$.

Lemma 27 (see [21]). Let $E$ be a Banach space. If $H \subseteq C([0$, $T] ; E)$ is equicontinuous and $x_{0} \in C([0, T] ; E)$, then $\bar{c} \bar{o}(H \cup$ $\left.\left\{x_{0}\right\}\right)$ is also equicontinuous in $C([0, T] ; E)$.

Lemma 28 (see [40]). Let $H$ be a bounded subset of E. Then, for each $\varepsilon>0$, there exists a sequence $\left\{u_{n}\right\} \subseteq H$ such that

$$
\alpha(H) \leq 2 \alpha\left(\left\{u_{n}\right\}\right)+\varepsilon .
$$

Lemma 29 (see [41], Lemma 2.7). For each $n \in N, 0<\lambda<1$, and $T>0$, we put

$$
S_{n}=\left[\lambda^{n}+C_{n}^{1} \lambda^{n-1} T+C_{n}^{2} \lambda^{n-2} \frac{T^{2}}{2 !}+\cdots+\frac{T^{n}}{n !}\right] .
$$

Then, $\lim _{n \rightarrow \infty} S_{n}=0$.

Before we proceed further, we present the following useful lemma.

Lemma 30. Let $E$ be a Banach space and $X$ be a subset of $E$. Let $f: X \longrightarrow E$ be a mapping such that there exists a nonnegative constant $\gamma \geq 0$ satisfying

$$
\alpha(f(\Delta)) \leq \gamma \alpha(\Delta),
$$

for any countable subset $\Delta$ of $X$. Then, for any subset $M$ of $X$, we have

$$
\alpha(f(M))) \leq 2 \gamma \alpha(M) .
$$

Proof. Let $M$ be a subset of $X$ and $\varepsilon>0$ be fixed. In view of Lemma 28, there is a sequence $\left\{y_{n}\right\}$ of $f(M)$ such that

$$
\alpha(f(M)) \leq 2 \alpha\left(\left\{y_{n}\right\}\right)+\varepsilon .
$$

Further, for each $n \in N$, there is $x_{n} \in M$ such that $y_{n}=f$ $\left(x_{n}\right)$ and so

$$
\alpha\left(\left\{y_{n}\right\}\right)=\alpha\left(f\left(\left\{x_{n}\right\}\right)\right) \leq \gamma \alpha\left(\left\{x_{n}\right\}\right) \leq \gamma \alpha(M) .
$$

Linking (83) and (84), we obtain

$$
\alpha(f(M)) \leq 2 \gamma \alpha(M)+\varepsilon .
$$

The arbitrariness of $\varepsilon$ yields that

$$
\alpha(f(M)) \leq 2 \gamma \alpha(M) .
$$

If we replace the Kuratowski measure of noncompactness $\alpha$ by the Hausdorff measure of noncompactness $\chi$ in Lemma 30 , we obtain the following result.

Lemma 31. Let $E$ be a Banach space and $X$ be subset of $E$. Let $f: X \longrightarrow E$ be a mapping such that there exists a nonnegative constant $\gamma \geq 0$ satisfying

$$
\chi(f(\Delta)) \leq \gamma \chi(\Delta),
$$

for any countable subset $\Delta$ of $X$. Then, for any subset $M$ of $X$, we have

$$
\chi(f(M))) \leq \gamma \chi(M) .
$$

Proof. By virtue of [42], Lemma 2.9, the reasoning in the proof of Lemma 30 yields the desired result. result.

Now, we are in a position to state the following existence

Theorem 32. Let $f: I \times \mathscr{B} \times \Omega \rightarrow$ E and $\eta: \Omega \longrightarrow$ E be mappings satisfying the following assumptions:

(i) For each $\omega \in \Omega, f(. . ., \omega)$ is uniformly continuous on $I \times \mathscr{B}$

(ii) For each $(t, x) \in I \times \mathscr{B}, f(t, x$,. $)$ is measurable

(iii) There exists a function $C: I \times \Omega \longrightarrow R^{+}$such that

$$
\alpha(f(t, D, \omega))) \leq C(t, \omega) \alpha(D),
$$

for all $\omega \in \Omega, t \in I$, and for any countable subset $D$ of $\mathscr{B}$, where each $C(., \omega) \in L^{1}\left([0, T] ; R^{+}\right)$ 
(iv) $m=\sup \{\|f(t, x, \omega)\| ; t \in I, x \in \mathscr{B}, \omega \in \Omega\}<+\infty$,

(v) $\eta$ is measurable and

$$
\sup \left\{\left\|\eta(\omega)-x_{0}\right\|: \omega \in \Omega\right\}=r_{0}<r
$$

Then, there exists a random solution of (77) on $\left[0, T_{1}\right]$ $\times \Omega$, with $T_{1}=\min \left\{T, r-r_{0} / m\right\}$.

Proof. Let

$$
\begin{aligned}
K & =\left\{u \in \mathscr{C}\left(I_{1}, E\right) ;\|u(s)-u(t)\|\right. \\
& \left.\leq m|s-t|, ? \text { and } ?\left\|u(t)-x_{0}\right\| \leq r, \forall s, t \in I_{1}\right\},
\end{aligned}
$$

where $I_{1}=\left[0, T_{1}\right]$. Clearly, $K$ is a nonempty bounded closed convex equicontinuous subset of $C(I 1 ; E)$ and $u(t) \in \mathscr{B}$ for each $(u, t) \in K \times I_{1}$.

Define the mapping $W: \Omega \times K \longrightarrow K$ by

$$
W(\omega, u)(t)=\eta(\omega)+\int_{0}^{t} f(s, u(s), \omega) d s
$$

for all $\omega \in \Omega, u \in K$, and $t \in I_{1}$. We show that $W$ is a random operator. To see this, let $u \in K$ be fixed. Since the mapping $\omega \longrightarrow f(s, u(s), \omega)$ is measurable for each $s \in I_{1}$ and the mapping $s \longrightarrow f(s, u(s), \omega)$ is continuous for each $\omega \in \Omega$, then the mapping $F_{u:} I_{1} \times \Omega \longrightarrow E,(s, \omega) \longrightarrow f(s, u(s), \omega)$ satisfies the condition $(C, \Omega)$. Thus, by Proposition 24, the mapping $\widehat{F}_{u}: \Omega \rightarrow \mathscr{C}\left(I_{1} ; E\right)$ defined by $\widehat{F}_{u}(\omega)=F_{u}(\omega,$.$) for every \omega$ $\in \Omega$ is measurable. Further, observe that mapping

$$
L: \mathcal{C}\left(I_{1} ; E\right) \rightarrow \mathcal{C}\left(I_{1} ; E\right) \text { defined by } L(v)(t)=\int_{0}^{t} v(s) d s
$$

for all $\mathrm{v} \in \mathcal{C}\left(I_{1} ; E\right)$, and $t \in I_{1}$ is linear and hence, by [1], Theorem $2.14, L \circ \widehat{F}_{u}$ is measurable. Therefore, $W(., u)=\eta+L \circ$ $\widehat{F}_{u}$ is measurable. The arbitrariness of $u \in K$ yields that $W$ is a random operator. Now, we will prove that $W$ fulfills all conditions of Theorem 7. To achieve this, let $\omega \in \Omega$ be fixed. First, by (i) for $\varepsilon>0$, there exists $\delta(\omega)>0$ such that

$$
\left\|f\left(t_{1}, x_{1}, \omega\right)-f\left(t_{2}, x_{2}, \omega\right)\right\|<\frac{\varepsilon}{T_{1}},
$$

for all $\left(t_{1}, x_{1}\right),\left(t_{2}, x_{2}\right) \in I_{1} \times \mathscr{B}$ satisfying $\left\|x_{1}-x_{2}\right\|<\delta(\omega)$ and $\left|t_{1}-t_{2}\right|<\delta(\omega)$. Let $u, v \in K$ such that

$$
\|v-u\|_{\infty}=\sup _{t \in I_{1}}\|v(t)-u(t)\|<\delta(\omega)
$$

We obtain

$$
\begin{aligned}
& \|W(\omega, v)(t)-W(\omega, u)(t)\| \\
& \quad \leq \int_{0}^{t}\|f(s, v(s), \omega)-f(s, u(s), \omega)\| d s \\
& \quad \leq \frac{\varepsilon}{T_{1}} t<\varepsilon,
\end{aligned}
$$

for each $t \in I_{1}$. Hence,

$$
\|W(\omega, v)-W(\omega, u)\|_{\infty}<\varepsilon
$$

Thus, $W(\omega,$.$) is uniformly continuous and, therefore, by$ arbitrariness of $\omega, W$ is uniformly continuous. Next, we claim that $W$ is countably $\alpha_{C}$-convex-power condensing. Let $H$ be a countable subset of $K$ and let $u \in H$. We see that for $\varepsilon>0$ there exists $\delta(\omega)>0$ such that

$$
\|f(s, u(s), \omega)-f(t, u(t), \omega)\|<\varepsilon,
$$

for all $(s, t) \in I_{1}^{2}$ satisfying $|s-t|<\delta(\omega) / \max \{1, m\}$. Then, $\|f(s, u(s), \omega)-f(t, u(t), \omega)\| \rightarrow 0$ as $t \rightarrow s$ independently to $u \in H$.

Hence, $\{f(., u(),. \omega): u \in H\}$ is equicontinuous. By using Lemma 26 and assumption (iii), we obtain for each $t \in I_{1}$

$$
\begin{aligned}
\alpha(W(\omega, H)(t)) & =\alpha\left(\left\{\eta(\omega)+\int_{0}^{t} f(s, u(s), \omega) d s: u \in H\right\}\right) \\
& \leq \int_{0}^{t} \alpha(f(s, H(s), \omega)) d s \leq \int_{0}^{t} C(s, \omega) \alpha(H(s)) d s \\
& \leq\left[\int_{0}^{t} C(s, \omega) d s\right] \alpha_{C}(H) .
\end{aligned}
$$

Let $0<\lambda<1$, we know that there is a continuous function $\varphi(\omega): \quad I_{1} \rightarrow \mathbb{R}^{+}\left(\right.$with $\left.M(\omega)=1 / 2 \max \left\{|\varphi(\omega)(t)|: t \in I_{1}\right\}\right)$ such that

$$
\int_{0}^{T}|C(s, \omega)-\varphi(\omega)(s)| d s<\frac{\lambda}{2}
$$

So, for each $t \in I_{1}$, we have

$$
\begin{aligned}
\alpha(W(\omega, H)(t)) \leq & {\left[\left(\int_{0}^{t}|C(s, \omega)-\varphi(\omega)(s)| d s\right.\right.} \\
& \left.\left.+\int_{0}^{t}|\varphi(\omega)(s)| d s\right)\right] \alpha_{C}(H) \\
\leq & (\lambda+M(\omega) t) \alpha_{C}(H) .
\end{aligned}
$$

Thus,

$$
\alpha\left(W^{\left(1, \theta_{0}\right)}(\omega, H)(t)\right) \leq(\lambda+M(\omega) t) \alpha_{\mathcal{C}}(H), \text { for each } t \in I_{1},
$$


where $\theta_{0} \in K$ is the mapping defined by $\theta_{0}(t)=x_{0}$ for every $t \in I_{1}$.

Now, since for $n \geq 1, \bar{c} \bar{o}\left(W^{\left(n, \theta_{0}\right)}(\omega, H) \cup\left\{\theta_{0}\right\}\right) \subset K$, then $f\left(., \bar{c} \bar{o}\left(W^{\left(n, \theta_{0}\right)}(\omega, H) \cup\left\{\theta_{0}\right\}\right)(),. \omega\right)$ is equicontinious. Thus, in view of (101), assumption (iii) and Lemmas 26-30, we obtain

$$
\begin{aligned}
\alpha\left(W^{\left(2, \theta_{0}\right)}(\omega, H)(t)\right) & =\alpha\left(W\left(\omega, \bar{c} \bar{o}\left(W^{\left(1, \theta_{0}\right)}\right)(\omega, H) \cup\left\{\theta_{0}\right\}(t)\right)\right) \\
& \leq \alpha\left(\int_{0}^{t} f\left(., \bar{c} \bar{o}\left(W^{\left(1, \theta_{0}\right)}(\omega, H) \cup\left\{\theta_{0}\right\}\right)(.), \omega\right)(s) d s\right) \\
& \left.\leq \int_{0}^{t} 2 C(s, \omega) \alpha\left(\bar{c} \bar{o}\left(W(\omega, H) \cup\left\{\theta_{0}\right\}\right)\right)(s)\right) d s \\
& \leq 2 \int_{0}^{t}(|C(s, \omega)-\varphi(\omega)(s)|+|\varphi(\omega)(s)|) \\
& (\lambda+M(\omega) s) \alpha_{c}(H) d s \\
& \leq\left[(\lambda+M(\omega) t) \lambda+M(\omega)\left(\lambda t+\frac{M(\omega) t^{2}}{2}\right)\right] \alpha_{C}(H) \\
& =\left(\lambda^{2}+2 \lambda(M(\omega) t)+\frac{(M(\omega) t)^{2}}{2}\right) \alpha_{C}(H) .
\end{aligned}
$$

Hence, by mathematical induction, for all $n \geq 1$ and $t \in I_{1}$ , we obtain

$$
\alpha\left(W^{\left(n, \theta_{0}\right)}(\omega, H)(t)\right) \leq \Gamma_{n}(t) \alpha_{C}(H)
$$

where $\Gamma_{n}(t)=\lambda^{n}+C_{n}^{1} \lambda^{n-1}(M(\omega) t)+C_{n}^{2} \lambda^{n-2}\left((M(\omega) t)^{2} / 2 !\right)$ $+\cdots+\left((M(\omega) t)^{n} / n !\right)$.

Using the equicontinuity of the $W^{\left(n, \theta_{0}\right)}(\omega, H)$ on $I_{1}$, we get

$$
\alpha_{C}\left(W^{\left(n, \theta_{0}\right)}(\omega, H)\right) \leq \Gamma_{n}\left(T_{1}\right) \alpha_{C}(H)
$$

Since $0<\lambda<1$ and $M(\omega) T_{1}>0$, Lemma 29 yields that there exists a positive integer $n_{0}(\omega)$ such that $\Gamma_{n_{0}(\omega)}\left(T_{1}\right)<1$ . Consequently,

$$
\alpha_{C}\left(W^{\left(n_{0}(\omega), \theta_{0}\right)}(\omega, H)\right)<\alpha_{C}(H)
$$

for any countable subset $H$ of $K$ with $\alpha_{C}(H)>0$. Thus, $W($ $\omega,$.$) is countably \alpha_{C}$-convex-power condensing about $\theta_{0}$ and $n_{0}(\omega)$. Therefore, $W$ is countably $\alpha_{C}$-convex-power condensing. Now, Theorem 7 guarantees that $W$ has a random fixed point $\xi: \Omega \longrightarrow K \subset \mathcal{C}\left(I_{1} ; E\right)$. By Proposition 24 , the mapping $\xi$ satisfies condition $(\mathcal{C}, \Omega)$ as a mapping of $I_{1} \times \Omega$ into $E$. Further, by (92), $\xi$ satisfies condition $\left(C^{1}, \Omega\right)$ and $\xi$ is a random solution of problem (77).

As a convenient specialization of Theorem 32, we consider the particular case when $\eta=0$ and $x_{0}=0$. We, therefore, obtain the following result.

Corollary 33. Let $f: I \times \mathcal{B} \times \Omega \longrightarrow E$ be a mapping satisfying the following assumptions: (i) For each $\omega \in \Omega, f(., ., \omega)$ is uniformly continuous on $I \times B$

(ii) For each $(t, x) \in I \times B, f(t, x$,. $)$ is measurable

(iii) There exists a function $C: I \times \Omega \rightarrow \mathbb{R}^{+}$such that

$$
\alpha(f(t, D, \omega))) \leq C(t, \omega) \alpha(D),
$$

for all $\omega \in \Omega, t \in I$, and for any countable subset $D$ of $\mathscr{B}$, where each $C(., \omega) \in L^{1}\left([0, T] ; \mathbb{R}^{+}\right)$

(vi) $m=\sup \{\|f(t, x, \omega)\| ; t \in I, x \in B, \omega \in \Omega\}<+\infty$,

Then, the random differential equation

$$
\forall(t, \omega) \in I \times \Omega: \frac{d}{d t} u(t, \omega)=f(t, u(t, \omega), \omega), u(0, \omega)=0
$$

has a random solution on $\left[0, T_{1}\right] \times \Omega$, with $T_{1}=\min \{T, r / m\}$.

Remark 34.

(1) Theorem 32 improves [11], Theorem 4.3. In our considerations, mapping $C$ is not subject to any restriction

(2) Theorem 32 remains true if we replace the Kuratowski measure of noncompactness by the Hausdorff measure of noncompactness

In the following, we present a new approach to solve (77) based on Theorem 19. To achieve this, let $E$ be an ordered separable real Banach space with a normal cone $P$ and $\beta$ be the De Blasi measure of weak noncompactness on $E$ and on $C(I ; E)$.

Theorem 35. Let $f: I \times E \times \Omega \longrightarrow E$ be a mapping satisfying the following assumptions:

(i) For each $\omega \in \Omega, f(., ., \omega)$ is continuous on $I \times E$ and maps bounded sets into bounded ones

(ii) For all $t \in I$ and $x \in E, f(t, x,$.$) is measurable$

(iii) There exist two mappings $v_{0}, w_{0}: I \times \Omega \longrightarrow E$ satisfying condition $\left(C^{1}, \Omega\right)$ and the following conditions hold

$$
\begin{aligned}
\forall \omega \in \Omega, t \in I, \frac{d v_{0}}{d t}(t, \omega) & \leq f\left(t, v_{0}(t, \omega), \omega\right), f\left(t, w_{0}(t, \omega), \omega\right) \\
& \leq \frac{d w_{0}}{d t}(t, \omega),
\end{aligned}
$$

(iv) There is a measurable mapping $M: \Omega \longrightarrow R^{+} \backslash\{0\}$ satisfying 


$$
f(t, y, \omega)-f(t, x, \omega) \geq-M(\omega)(y-x),
$$

whenever $v_{0}(t, \omega) \leq x \leq y \leq w_{0}(t, \omega),(t, \omega) \in I \times \Omega$

(v) there is a mapping $\rho: \Omega \longrightarrow[0,+\infty)$ such that for each $\omega \in \Omega$, for any monotone sequence $V=\left\{u_{n}\right\}$ of $\left[v_{0}(., \omega), w_{0}(., \omega)\right]$ and for all $a, b \in I$ with $a \leq b$ we have

$$
\beta(f([a, b] \times V \times\{\omega\})) \leq \rho(\omega) \beta(V([a, b])),
$$

where $f([a, b] \times V \times\{\omega\}):=\left\{f\left(s, u_{n}(s), \omega\right) ; s \in[a, b], n \geq 1\right\}$

Then, the random differential equation (77) has a random solution on $I \times \Omega$ which can obtained by monotone iterative procedure starting from $v_{0}$ or from $w_{0}$.

Remark 36. Set $g(t, x, \omega)=f(t, x, \omega)+M(\omega) x$, for $(t, x, \omega)$ $\in I \times E \times \Omega$. Then,

$\left(\mathrm{G}_{1}\right)$ For each $(t, \omega) \in I \times \Omega$,

$$
g(t, x, \omega) \leq g(t, y, \omega) \text { whenever } v_{0}(t, \omega) \leq x \leq y \leq w_{0}(t, \omega)
$$

$\left(\mathrm{G}_{2}\right)$ For each $\omega \in \Omega$, for any monotone sequence $V=\{$ $\left.u_{n}\right\}$ in $\left[v_{0}(., \omega), w_{0}(., \omega)\right]$ and for all $a, b \in I$ with $a \leq b$, we have

$$
\beta(g([a, b] \times V \times\{\omega\})) \leq \gamma(\omega) \beta(V([a, b])),
$$

where $\gamma(\omega)=\rho(\omega)+M(\omega)$

Proof. The random problem (77) is equivalent to the random problem

$$
\begin{gathered}
\forall(t, \omega) \in I \times \Omega: \frac{d}{d t} u(t, \omega)+M(\omega) u(t, \omega) \\
=g(t, u(t, \omega), \omega), u(0, \omega)=\eta(\omega)
\end{gathered}
$$

which is equivalent to the random problem

$$
\begin{aligned}
& \forall(t, \omega) \in I \times \Omega: \frac{d}{d t}\left(e^{M(\omega) t} u(t, \omega)\right) \\
& \quad=e^{M(\omega) t} g(t, u(t, \omega), \omega), u(0, \omega)=\eta(\omega) .
\end{aligned}
$$

Let us write (115) as a random integral equation

$$
\begin{aligned}
\forall(t, \omega) \in I \times \Omega: u(t, \omega)= & e^{-M(\omega) t} \eta(\omega) \\
& +\int_{0}^{t} e^{-M(\omega)(t-s)} g(s, u(s, \omega), \omega) d s
\end{aligned}
$$

Define the mapping $\Phi: \Omega \times \mathcal{C}(I, E) \longrightarrow \mathcal{C}(I, E)$ by

$$
\Phi(\omega, u)(t)=e^{-M(\omega) t} \eta(\omega)+\int_{0}^{t} e^{-M(\omega)(t-s)} g(s, u(s), \omega) d s
$$

for each $(t, \omega) \in I \times \Omega$ and any $u \in \mathcal{C}(I, E)$

We will show that $\Phi$ is a random operator. To this end, let $u \in \mathcal{C}(I, E)$ be fixed. Notice first that for each $t \in I$, the mapping $\Omega \longrightarrow[0,1], \omega \longrightarrow e^{-M(\omega) t}$ is measurable, since $\exp : \mathbb{R} \longrightarrow \mathbb{R}^{+} \backslash\{0\}, x \longrightarrow e^{x}$ is continuous and $M$ is measurable. Let $t \in I$ and $s \in[0, t]$. Using Lemma 8 together with assumptions (ii) and (iv) we infer that $\omega \longrightarrow e^{-M(\omega) t} \eta(\omega)$ and $\omega \longrightarrow e^{-M(\omega)(t-s)} g(s, u(s), \omega)$ are measurable. Furthermore, since $s \longrightarrow e^{-M(\omega)(t-s)} g(s, u(s), \omega)$ is continuous for each $\omega \in \Omega$, then the mapping $G_{u, t}:[0, t] \times \Omega \longrightarrow E,(s, \omega)$ $\longrightarrow e^{-M(\omega)(t-s)} g(s, u(s), \omega)$ satisfies the condition $(C, \Omega)$. Thus, by Proposition 24, the mapping $\widehat{G}_{u, t}: \Omega \rightarrow \mathscr{C}([0, t] ; E$ ) defined by $\widehat{G}_{u, t}(\omega)=G_{u, t}(\omega,$.$) for every \omega \in \Omega$, is measurable. Next, observe that the mapping $L_{t}: \mathcal{C}([0, t] ; E) \longrightarrow \mathcal{C}($ $[0, t] ; E)$ defined by $L_{t}(v)(\tau)=\int_{0}^{\tau} v(s) d s$ for all $v \in \mathscr{C}(I ; E)$ and $\tau \in[0, t]$ is linear. Hence, by [1], Theorem 2.14, $L_{t} \circ \widehat{G}_{u, t}$ is measurable. By virtue of Proposition 24, we see that the mapping $(\omega, \tau) \mapsto L_{t} \circ \widehat{G}_{u, t}(\omega)(\tau)$ satisfies condition $(C, \Omega)$.

Accordingly, for each $\tau \in[0, t]$, the mapping $\omega \mapsto L_{t}$ 。 $\widehat{G}_{u, t}(\omega)(\tau)=\int_{0}^{\tau} e^{-M(\omega)(t-s)} g(s, u(s), \omega) d s$ is measurable. In particular, the mapping $\omega \mapsto L_{t} \circ \widehat{G}_{u, t}(\omega)(\tau)=\int_{0}^{\tau} e^{-M(\omega)(t-s)} g$ $(s, u(s), \omega) d s$ is measurable.

Therefore,

$\omega \mapsto \Phi(\omega, u)(\tau)=e^{-M(\omega)(t)} \eta(\omega)+\int_{0}^{\tau} e^{-M(\omega)(t-s)} g(s, u(s), \omega) d s$,

is measurable. This is true for each $t \in I$. By a simple verification using assumption (i), we can show that $t \mapsto \Phi(\omega, u)(t)$ $=e^{-M(\omega) t} \eta(\omega)+\int_{0}^{t} e^{-M(\omega)(t-s)} g(s, u(s), \omega) d s$ is continuous for each $\omega \in \Omega$. Then, the mapping $(\omega, t) \mapsto \Phi(\omega, u)(t)$ satisfies condition $(C, \Omega)$. Finally by invoking Proposition 24, we conclude that $\Phi(., u): \Omega \longrightarrow \mathcal{C}(I, E)$ is measurable. The arbitrariness of $u \in \mathcal{C}(I, E)$ yields that $\Phi$ is a random operator.

Now, we will prove that $\Phi$ fulfills all conditions of Theorem 19. This will be achieved in three steps:

Step 1. We show that $\Phi$ is continuous. To this end, fix $\omega \in \Omega$ and $u \in C(I, E)$. Let $\left\{u_{n}\right\}$ be a sequence in $C(I, E)$ which converges to $u$. Then, there is an integer $N \in \mathbb{N}$ such that

$$
\left\|u_{n}-u\right\|_{\infty}<1 \text { whenever } n \geq N
$$

Hence,

$$
\left\|u_{n}\right\|_{\infty} \leq\|u\|_{\infty}+1=r \text { for each } n \geq N
$$


Now, we put

$$
F_{\omega, n}(s)=g\left(s, u_{n}(s), \omega\right), F_{\omega}(s)=g(s, u(s), \omega)
$$

and

$$
K_{\omega}=\sup \{\|g(s, z, \omega)\| ; s \in[0, T] \text { and }\|z\| \leq r\}<\infty
$$

Note that, for all $n \geq N$ and $s \in[0, T]$ we have $\left\|F_{\omega, n}(s)\right\|$ $\leq K_{\omega}$. Furthermore, the continuity of $g(., ., \omega)$ on $I \times E$ implies that

$$
\left\|F_{\omega, n}(s)-F_{\omega}(s)\right\| \rightarrow 0 \text { as } n \rightarrow \infty
$$

The dominated convergence theorem yields

$$
\int_{0}^{T}\left\|F_{\omega, n}(s)-F_{\omega}(s)\right\| d s \rightarrow 0 \text { as } n \rightarrow \infty .
$$

On the other hand, for each $t \in[0, T]$,

$$
\begin{aligned}
\left\|\Phi\left(\omega, u_{n}\right)(t)-\Phi(\omega, u)(t)\right\| & \leq \int_{0}^{t}\left\|g\left(s, u_{n}(s), \omega\right)-g(s, u(s), \omega)\right\| d s \\
& \leq \int_{0}^{T}\left\|F_{\omega, n}(s)-F_{\omega}(s)\right\| d s .
\end{aligned}
$$

Hence,

$$
\left\|\Phi\left(\omega, u_{n}\right)-\Phi(\omega, u)\right\|_{\infty} \leq \int_{0}^{T}\left\|F_{\omega, n}(s)-F_{\omega}(s)\right\| d s .
$$

Therefore, $\left\|\Phi\left(\omega, u_{n}\right)-\Phi(\omega, u)\right\|_{\infty} \rightarrow 0$ as $n \rightarrow \infty$ and consequently $\Phi(\omega,$.$) is continuous at u$. The arbitrariness of $u$ implies that $\Phi(\omega,$.$) is continuous on \mathcal{C}(I, E)$.

Step 2. We illuminate that $\Phi$ is increasing on the random interval

$\left[v_{0}, w_{0}\right]=\left\{x: \Omega \rightarrow \mathscr{C}(I, E): v_{0}(., \omega) \leq x(\omega) \leq w_{0}(., \omega)\right.$ for each $\left.\omega \in \Omega\right\}$.

To do this, let $\omega \in \Omega$ be fixed and $u, v \in C(I, E)$ with $v_{0}$. $, \omega) \leq u \leq v \leq w_{0}(., \omega)$. Using $\left(\mathrm{G}_{1}\right)$ we obtain

$$
g(t, u(t), \omega) \leq g(t, v(t), \omega) \text { for each } t \in I .
$$

Now, (117) becomes

$$
\Phi(\omega, u)(t) \leq \Phi(\omega, v)(t) \text { for each } t \in I
$$

Thus,

$$
\Phi(\omega, u) \leq \Phi(\omega, v)
$$

Therefore, $\Phi$ is increasing on $\left[v_{0}, w_{0}\right]$.
Step 3. We show that the following conditions hold:

$$
\forall \omega \in \Omega, v_{0}(., \omega) \leq \Phi\left(\omega, v_{0}(., \omega)\right), \Phi\left(\omega, w_{0}(., \omega)\right) \leq w_{0}(., \omega)
$$

To this end, let $\omega \in \Omega$. We begin by setting

$$
\begin{aligned}
& h_{1}(t, \omega)=\frac{d v_{0}}{d t}(t, w)+M(\omega) v_{0}(t, \omega), \\
& h_{2}(t, \omega)=\frac{d w_{0}}{d t}(t, w)+M(\omega) w_{0}(t, \omega) .
\end{aligned}
$$
have

Clearly, $h_{1}(., \omega), h_{2}(., \omega) \in \mathrm{C}(I, E)$ and for each $t \in I$, we

$$
\begin{aligned}
h_{1}(t, \omega) & =\frac{d v_{0}}{d t}(t, \omega)+M(\omega) v_{0}(t, \omega) \\
& \leq f\left(t, v_{0}(t, \omega), \omega\right)+M(\omega) v_{0}(t, \omega) \\
& =g\left(t, v_{0}(t, \omega), \omega\right) \leq g\left(t, \omega_{0}(t, \omega), \omega\right) \\
& =f\left(t, w_{0}(t, \omega), \omega\right)+M(\omega) w_{0}(t, \omega) \\
& \leq \frac{d w_{0}}{d t}(t, \omega)+M(\omega) \omega_{0}(t, \omega)=h_{2}(t, \omega) .
\end{aligned}
$$

Keeping in mind the fact that $(d / d t)\left(e^{M(\omega) t} v_{0}(t, \omega)\right)=$ $e^{M(\omega) t} h_{1}(t, \omega)$ and $(d / d t)\left(e^{M(\omega) t} w_{0}(t)\right)=e^{M(\omega) t} h_{2}(t, \omega)$, we deduce that for each $t \in I$ have:

$$
\begin{aligned}
e^{M(\omega) t} v_{0}(t, \omega) & =v_{0}(0, \omega)+\int_{0}^{t} e^{M(\omega) s} h_{1}(s, \omega) d s \\
& \leq \eta(\omega)+\int_{0}^{t} e^{M(\omega) s} g\left(t, v_{0}(s, \omega), \omega\right) d s \\
& \leq \eta(\omega)+\int_{0}^{t} e^{M(\omega) s} g\left(t, w_{0}(s, \omega), \omega\right) d s \\
& \leq w_{0}(0, \omega)+\int_{0}^{t} e^{M(\omega) s} h_{2}(s, \omega) d s \\
& =e^{M(\omega) t} w_{0}(t, \omega) .
\end{aligned}
$$
$w_{0}(., \omega)$.

Accordingly, $\quad v_{0}(., \omega) \leq \Phi\left(\omega, v_{0}(., \omega)\right) \leq \Phi\left(\omega, w_{0}(., \omega)\right) \leq$

Step 4. The reasoning in [39], Theorem 3.2 yields that each $\Phi(\omega,$.$) satisfies the condition \left(\mathscr{P}\left(n_{0}\right)\right)$. Therefore, the random operator $\Phi$ satisfies the condition $\left(\mathscr{P}\left(n_{0}\right)\right)$.

Now, by applying Theorem 19 , we infer that $\Phi$ has a random fixed point $\xi$ which can obtained by monotone iterative procedure starting from $v_{0}\left(\right.$ or $\left.w_{0}\right): \Omega \longrightarrow \mathcal{C}(I, E)$. The mapping $\widehat{\xi}: I \times \Omega \longrightarrow E$ defined by $\widehat{\xi}(t, \omega)=\xi(\omega)(t)$ is measurable as mapping from $\Omega$ into $\mathscr{C}(I, E)$. Hence, by Proposition 24, it satisfies the condition $(C, \Omega)$. Further, $\xi_{b}$ satisfies the random integral equation (116). Then, for every $\omega \in \Omega$, we have $\widehat{\xi}(., \omega) \in \mathcal{C}^{1}(I, E)$ and so $\widehat{\xi}$ satisfies condition $\left(\mathscr{C}^{1}, \Omega\right) . \xi$ is a random solution of problem (77). 


\section{Data Availability}

The authors received no data support for this research.

\section{Conflicts of Interest}

The authors declare that they have no conflicts of interest.

\section{References}

[1] A. T. Bharucha-Reid, "Random integral equations," in Mathematics in Science and Engineering, vol. 96, Academic Press, New York-London, 1972.

[2] A. C. H. Lee and W. J. Padgett, "Random contractors with random nonlinear majorant functions," Nonlinear Analysis: Theory, Methods \& Applications, vol. 3, no. 5, pp. 707-715, 1979.

[3] W. J. Padgett, "Almost surely continuous solutions of a nonlinear stochastic integral equation," Mathematical systems theory, vol. 10, no. 1, pp. 69-75, 1976.

[4] W. J. Padgett and C. P. Tsokos, "On a semi-stochastic model arising in a biological system," Mathematical Biosciences, vol. 9, pp. 105-117, 1970.

[5] W. J. Padgett and C. P. Tsokos, "Random integral equations with applications to stochastic sytems," in Lecture Notes in Mathematics, vol. 233, Springer-Verlag, Berlin-New York, 1971.

[6] W. J. Padgett and C. P. Tsokos, "On a stochastic integral equation of the Volterra type in telephone traffic theory," Journal of Applied Probability, vol. 8, no. 2, pp. 269-275, 1971.

[7] W. J. Padgett and C. P. Tsokos, "Random integral equations with applications to life sciences and engineering," in Mathematics in Science and Engineering, vol. 108, Academic Press [A subsidiary of Harcourt Brace Jovanovich, Publishers], New Yor-London, 1974.

[8] W. J. Padgett and C. P. Tsokos, "On the existence of a unique solution of a stochastic integral equation in hereditary mechanics," Journal of mathematical and physical sciences, vol. 8, pp. 299-308, 1974.

[9] B. L. S. Prakasa Rao, "Stochastic integral equations of mixed type. II," Journal of mathematical and physical sciences, vol. 7, pp. 245-260, 1973.

[10] A. T. Bharucha-Reid, "Fixed point theorems in probabilistic analysis," Bulletin of the American Mathematical Society, vol. 82, no. 5, pp. 641-658, 1976.

[11] S. Itoh, "Random fixed point theorems with an application to random differential equations in Banach spaces," Journal of Mathematical Analysis and Applications, vol. 67, no. 2, pp. 261-273, 1979.

[12] S. Reich, "A random fixed-point theorem for set-valued mappings," Atti della Accademia Nazionale dei Lincei. Classe di Scienze Fisiche, Matematiche e Naturali. Rendiconti, vol. 64, pp. 65-66, 1978.

[13] L. E. Rybiński, "Random fixed points and viable random solutions of functional-differential inclusions," Journal of mathematical analysis and applications, vol. 142, no. 1, pp. 53-61, 1989.

[14] V. M. Sehgal and S. P. Singh, "On random approximations and a random fixed point theorem for set valued mappings," Proceedings of the American Mathematical Society, vol. 95, no. 1, pp. 91-94, 1985.
[15] V. M. Sehgal and C. Waters, "Some random fixed point theorems for condensing operators," Proceedings of the American Mathematical Society, vol. 90, no. 3, pp. 425-429, 1984.

[16] N. S. Papageorgiou, "On the measurable selection approach in random differential inclusions, fixed point theory and optimization," Journal of Mathematical and Physical Sciences, vol. 24, no. 5, pp. 331-345, 1990.

[17] K. K. Tan and X. Z. Yuan, "Random fixed-point theorems and approximation in cones," Journal of Mathematical Analysis and Applications, vol. 185, no. 2, pp. 378-390, 1994.

[18] N. Hussain, "Random fixed points for $\psi$-contractions with application to random differential equations," Filomat, vol. 31, no. 3, pp. 759-779, 2017.

[19] B. N. Sadovskii, "On a fixed point principle," Functional Analysis and Its Applications, vol. 1, no. 2, pp. 74-76, 1967.

[20] A. El-Ghabi and M. A. Taoudi, "Random fixed point theorems under weak topology features and application to random integral equations with lack of compactness," Journal of Fixed Point Theory and Applications, vol. 22, no. 4, 2020.

[21] J. X. Sun and X. Y. Zhang, "A fixed point theorem for convexpower condensing operators and its applications to abstract semilinear evolution equations," Acta Mathematica Sinica,Chinese Series, vol. 48, no. 3, pp. 439-446, 2005.

[22] C. J. Himmelberg, "Measurable relations," Fundamenta Mathematicae, vol. 87, no. 1, pp. 53-72, 1975.

[23] R. P. Agarwal, N. Hussain, and M. A. Taoudi, "Fixed point theorems in ordered Banach spaces and applications to nonlinear integral equations," Abstract and Applied Analysis, vol. 2012, Article ID 245872, 15 pages, 2012.

[24] K. Ezzinbi and M. A. Taoudi, "Sadovskii-Krasnosel'skii type fixed point theorems in Banach spaces with application to evolution equations," Journal of Applied Mathematics and Computing, vol. 49, no. 1-2, pp. 243-260, 2015.

[25] K. Ezzinbi, S. Ghnimi, and M. A. Taoudi, "New MonchKrasnosel'skii type fixed point theorems applied to solve neutral partial integrodifferential equations without compactness," Journal of Fixed Point Theory and Applications, vol. 22, no. 3, 2020.

[26] N. Hussain, A. E. Al-Mazrooei, A. R. Khan, and J. Ahmad, "Solution of Volterra integral equation in metric spaces via new fixed point theorem," Univerzitet $u$ Nišu, vol. 32, no. 12, pp. 4341-4350, 2018.

[27] N. Hussain and M. A. Taoudi, "Fixed point theorems for multivalued mappings in ordered Banach spaces with application to integral inclusions," Fixed Point Theory and Applications, vol. 2016, no. 1, p. 9, 2016.

[28] N. Hussain and M. A. Taoudi, "Krasnosel'skii-type fixed point theorems with applications to Volterra integral equations," Fixed Point Theory and Applications, vol. 2013, no. 1, 2013.

[29] B. Matani, J. R. Roshan, and N. Hussain, “An extension of Darbo's theorem via measure of non-compactness with its application in the solvability of a system of integral equations," Univerzitet u Nišu, vol. 33, no. 19, pp. 6315-6334, 2019.

[30] J. Banaś and K. Goebel, "Measures of noncompactness in Banach spaces," in Lecture Notes in Pure and Applied Mathematics, vol. 60, Marcel Dekker, Inc.,, New York, 1980.

[31] J. Banaś and J. Rivero, "On measures of weak noncompactness," Annali di Matematica Pura ed Applicata, vol. 151, no. 1, pp. 213-224, 1988. 
[32] A. Chlebowicz and M. A. Taoudi, "Measures of weak noncompactness and fixed points," in Advances in nonlinear analysis via the concept of measure of noncompactness, pp. 247-296, Springer, Singapore, 2017.

[33] R. P. Agarwal, D. O'Regan, and M.-A. Taoudi, "Fixed point theorems for ws-compact mappings in Banach spaces," Fixed Point Theory and Applications, vol. 2010, no. 1, Article ID 183596, 2010.

[34] F. S. De Blasi, "On a property of the unit sphere in a Banach space," Bulletin mathématique de la Société des Sciences Mathématiques de la République Socialiste de Roumanie, vol. 21, no. 3-4, pp. 259-262, 1977.

[35] H. B. Shi Hong-bo, "The fixed point theorem of convex-power condensing operator and its applications in locally convex spaces," Journal of South west University (Natural Science Edition), vol. 29, no. 9, pp. 1-5, 2007.

[36] D. G. Luenberger, Optimization by Vector Space Methods, John Wiley \& Sons, Inc., New York-London-Sydney, 1969.

[37] K. Fan, "Extensions of two fixed point theorems of F. E. Browder," Mathematische zeitschrift, vol. 112, no. 3, pp. 234-240, 1969.

[38] A. Khchine, L. Maniar, and M. A. Taoudi, "Krasnosel'skii-type fixed point theorems for convex-power condensing mappings in locally convex spaces," Journal of Fixed Point Theory and Applications, vol. 19, no. 4, pp. 2985-3012, 2017.

[39] A. Alahmari, M. Mabrouk, and M. A. Taoudi, "Fixed point theorems for monotone mappings in ordered Banach spaces under weak topology features," Journal of Mathematics and Applications, vol. 42, pp. 5-19, 2019.

[40] D. Bothe, "Multivalued perturbations ofm-accretive differential inclusions," Israel Journal of Mathematics, vol. 108, no. 1, pp. 109-138, 1998.

[41] X. Zhang, L. Liu, and Y. Wu, "Global solutions of nonlinear second-order impulsive integro-differential equations of mixed type in Banach spaces," Nonlinear Analysis, vol. 67, no. 8, pp. 2335-2349, 2007.

[42] H. R. Henriquez, V. Poblete, and J. C. Pozo, "Mild solutions of non-autonomous second order problems with nonlocal initial conditions," Journal of Mathematical Analysis and Applications, vol. 412, no. 2, pp. 1064-1083, 2014. 